\title{
PRESTIGIO Y ESTIGMATIZACIÓN DE 60 NOMBRES PROPIOS EN 40 SUJETOS DE NIVEL EDUCACIONAL SUPERIOR
}

\author{
Prestige and stigmatization of 60 proper nouns in \\ 40 subjects of higher educational level (university) \\ Gastón Salamanca * \\ Lidia Pereira **
}

\begin{abstract}
RESUMEN
En este artículo presentamos los resultados obtenidos luego de aplicar una encuesta en que se indagaba la actitud de prestigio o estigmatización que 40 sujetos de nivel educacional superior (universitario) tenían respecto de 60 nombres propios. Los resultados muestran que sistemáticamente algunos nombres son asociados con prestigio/estrato social alto, y otros lo son con desprestigio/estrato social bajo. También se muestra que las respuestas relacionadas con estigmatización (nombres con los que se reirían y se asocian con estrato social bajo) son mucho más compactas que aquellas relacionadas con prestigio (nombres con los que serían populares y se asocian con estrato social alto), pues concentran cantidades similares de votos en menos nombres. Por último, se concluye que entre los sujetos encuestados hay una alta consciencia de la norma lingüística, especialmente entre los sujetos de sexo femenino.
\end{abstract}

Palabras clave: Sociolingüística, español de Chile, nombres propios.

\footnotetext{
* Facultad de Humanidades y Arte, Universidad de Concepción. Concepción, Chile. Correo electrónico: gaston. salamanca@gmail.com

** Licenciada en Educación con Mención en Español por la universidad de Concepción. Concepción, Chile. Correo electrónico: lidia.pereira@gmail.com

Artículo recibido el 16 de octubre de 2012. Aceptado el 26 de mayo de 2013.
} 


\begin{abstract}
In this paper we present the results obtained after applying a survey that probed the attitudes of prestige or stigma that 40 subjects of higher educational level (university) had about 60 names. The results show that some names are systematically related to prestige/high social stratum and others with lack of prestige/low social stratum. It also shows the answers related to stigmatization (names which may cause laughter and are associated to low social stratum) are more compact than the ones related to prestige (names with which they would be popular and are associated to high social stratum) because they concentrate similar number of votes in less names. Finally, we conclude that among the subjects surveyed there is a high awareness of the linguistic norm, especially among female subjects.
\end{abstract}

Keywords: Sociolinguistics, Chilean Spanish, proper nouns.

\title{
INTRODUCCIÓN
}

Uno de los conceptos que se ha relevado en sociolingüística es el de Actitud (Ver, por ejemplo, Fasold 1996). Este concepto se ha aplicado fundamentalmente a las lenguas y dialectos en contacto. Así, por ejemplo, se ha establecido que nuestra comunidad de habla estima que el inglés es una lengua prestigiosa y tiene respecto de ella actitudes positivas (Sáez, 1999). No ocurre así con nuestras lenguas vernáculas, respecto de las cuales se tiene, en general, actitudes que oscilan entre desconocimiento y discriminación. Una muestra de lo anterior ha sido descrita por Salas (1996), en su trabajo "Aymara mapuche ¿lenguas o dialectos?: En torno a la valoración social de los vernáculos chilenos". Aquí el autor enfatiza que los hablantes chilenos no dudan en considerar a lenguas como el inglés, el francés o el alemán "verdaderas" lenguas o idiomas, pero no tienen la misma convicción cuando se les pregunta, por ejemplo, si el mapudungun tiene ese estatus. De hecho, muchos de ellos lo consideran "sólo un dialecto".

En otro frente, Salamanca (2010) y Salamanca y Ramírez (2013) han relevado que nuestra sociedad tiene actitudes disímiles respecto de otras variantes del espańol. Por ejemplo, está instalada la idea de que los colombianos y peruanos "hablan bien" y no así los chilenos. Un caso interesante que estos autores relevan es la actitud que el chileno tiene respecto del español hablado en Argentina. 
Destacan, en este sentido, lo que denominan, siguiendo a Silva-Corvalán (2001), prestigio encubierto, y lo que han rotulado como permeabilidad asimétrica. Con ello dan cuenta de dos aspectos de esta relación: por una parte, el hecho de que, no obstante que los chilenos no verbalizan, en general, juicios de admiración respecto de "lo argentino", son receptivos a las influencias léxicas y de otro orden que tienen ese origen; y, por otra, el hecho de que esta receptividad de la variante chilena no tiene reciprocidad en la variante argentina.

Ahora, si consideramos las variaciones que se presentan dentro de un mismo dialecto, podemos observar que las actitudes también constituyen un aspecto prominente. En este sentido, trabajos como los de Valdivieso (1983), Tassara (1992), Salamanca y Valverde (2009) y Figueroa (2011) aportan a la discusión, pues destacan que al manipular alguna variable fonético-fonológica, cambian las percepciones y clasificaciones que los sujetos establecen respecto de sus pares.

El trabajo que presentamos se traslapa parcialmente con los señalados, pues se refiere a las actitudes que un grupo de sujetos tiene respecto de un aspecto de la lengua, pero no en un ámbito fonético-fonológico, sino con respecto a los nombres propios. Hasta donde sabemos, su único antecedente más directo es la tesis de Millán y Rodríguez (2011), quienes estudiaron el prestigio y estigmatización de 30 nombres propios en 40 sujetos con escaso nivel de escolaridad.

\section{MARCO DE REFERENCIA}

\subsection{Actitudes lingüisticas}

Los trabajos realizados por Valdivieso (1983) y Tassara (1992) han establecido que las variables sexo, edad y estrato sociocultural inciden en las actitudes lingüísticas. En efecto, los resultados obtenidos por estos investigadores, y que se condicen con los postulados generales de la sociolingüística, establecen que las mujeres son más sensibles a la norma lingüística y entregan juicios más categóricos en aspectos referidos al habla. Otra variable que incide es la edad, pues se ha establecido que los jóvenes realizan juicios más categóricos que los adultos. Por último, estos autores pudieron establecer que los sujetos pertenecientes a los estratos socioculturales más altos tienen mayor consciencia de la norma (socio) lingüística y emiten juicios más categóricos con respecto al uso de la lengua. 
También, la sociolingüística en general ha establecido que las urbes son más sensibles que las zonas rurales a la norma lingüística y las capitales más que las provincias.

\subsection{Algunos estudios sobre prestigiolestigmatización en el español de Chile}

Los estudios de Valdivieso y Tassara han establecido que los hablantes del español de Chile logran discriminar con un alto índice de acierto entre "locutores" que pertenecen a un estrato social determinado; al tiempo que las votaciones se concentran de manera bastante sistemática en aquellos locutores a los cuales contratarían con agrado y, sobretodo, en aquellos a los cuales no contratarían por ningún motivo.

Por su parte, en su trabajo de 2009, Salamanca y Valverde establecen que el rasgo fonético articulatorio anterior/posterior en las vocales del español de Chile se correlaciona con estrato sociocultural, de modo que si las vocales se anteriorizan, tienden a ser evaluadas por los sujetos como pertenecientes a un estrato sociocultural muy bajo ("flaite"), mientras que si se posteriorizan tienden a ser catalogadas como pertenecientes a sujetos de estrato sociocultural alto ("cuicos"). Figueroa (2011), por su parte, establece que en las consonantes africadas la prominencia del rasgo fricativo es asociado sistemáticamente con estrato sociocultural bajo, mientras que la prominencia del rasgo oclusivo es asociado sistemáticamente con estrato sociocultural alto o con ningún estrato sociocultural.

Finalmente, el estudio más reciente y que, como hemos dicho, se vincula directamente con el que aquí se realiza, es la tesis de Macarena Millán y Claudia Rodríguez, en el cual se indaga en el prestigio y estigmatización de 30 nombres propios en 40 sujetos de nivel educacional precario. Las principales conclusiones a las que arriban estas investigadoras son que:

- Las votaciones de las mujeres son las que concuerdan en mayor medida con las expectativas otorgadas por las encuestadoras en cuanto a los nombres propuestos. Esto corrobora lo constatado por investigaciones como las de Tassara (1992), las cuales sostienen que los jóvenes y las mujeres son dos grupos particularmente drásticos al momento de emitir juicios de valor con respecto a aspectos de la lengua.

- Hay una conciencia de norma de parte de los encuestados y encuestadas, aunque no en un nivel prominente. 
- Otro aspecto destacable es que, salvo en una pregunta, los votos se concentraban, en menos nombres, en las preguntas relativas a la estigmatización; es decir, existe una mayor cohesión en ese tipo de respuestas.

- Si consideramos la variable sexo, las mujeres presentan mayor cohesión que los hombres al valorar positiva y negativamente.

\section{METODOLOGÍA}

\subsection{Sujetos}

La muestra estuvo constituida por 40 estudiantes de primer año de fonoaudiología de la Universidad de Concepción, cuyas edades fluctuaban entre los 18 y 22 ańos. Esta cantidad de sujetos permite conformar una población suficientemente representativa y al mismo tiempo manejable para efectos del estudio.

\subsection{El instrumento}

Para obtener los datos, se diseñó una encuesta que contiene 3 ítems. En el primero de ellos, los sujetos debían pronunciarse respecto del prestigio o estigmatización de un nombre propio, su asociación con un estrato cultural alto o bajo, y seleccionar algunos nombres que les colocarían o no a sus hijos e hijas. En el segundo ítem, se pesquisaban los estereotipos que hay dentro de la sociedad chilena, en general, y dentro de la población juvenil, en particular, a través de una pregunta en que los encuestados debían imaginar las características de los sujetos portadores de determinados nombres (señalando sus posibles pasatiempos, el lugar en el que posiblemente vivirían, el tipo de colegio al que asistirían, etc.). Por último, se les solicitaba a los sujetos emitir su opinión con respecto a las características que a su juicio debía tener un nombre propio para que fuese considerado "bonito" o "feo".

Con el fin de no perder algunas observaciones relevantes en relación con el primer ítem, en este artículo presentamos los resultados correspondientes a esta sección de la encuesta. ${ }^{1}$

\footnotetext{
${ }^{1}$ En anexos se contiene la encuesta aplicada, en su versión para sujetos de sexo femenino y masculino, respectivamente.
} 


\subsection{Nombres seleccionados}

A continuación presentamos los nombres propios que los sujetos debían evaluar. Cabe señalar que inicialmente la lista fue considerablemente mayor (100 nombres) y fue propuesta por la coautora de este trabajo. Ahora, dado que esta cantidad de nombres se estimó muy extensa -lo cual podría repercutir negativamente en la disposición de los sujetos para responder la encuesta-, se decidió reducirla a 60, seleccionando para ello los nombres que se estimaron más representativos para las categorías "estigmatizado", "prestigioso” y "neutro”. Así, se buscó un consenso entre los autores del trabajo, el cual se produjo, entre otros, en los nombres que presentamos.

\subsubsection{Nombres masculinos}

\begin{tabular}{|c|c|c|}
\hline Estigmatizados & Prestigiosos & "Neutros" \\
\hline 1. Brian (Brayan) & 1. Cristóbal & 1. Rodrigo \\
\hline 2. Michael (Maickel) & 2. Sebastián & 2. Marcelo \\
\hline 3. Jason (Yeison) & 3. Martín & 3. Daniel \\
\hline 4. Jeremy (Yeremi) & 4. Alonso & 4. Andrés \\
\hline 5. Byron (Bairon) & 5. Tomás & 5. Claudio \\
\hline 6. Harry & 6. Ignacio & 6. Alejandro \\
\hline 7. Malcom & 7. Joaquín & 7. Pablo \\
\hline 8. Jordan & 8. Agustín & 8. Héctor \\
\hline 9. Kevin & 9. León & 9. Mauricio \\
\hline 10. Justin (Yastin) & 10. Máximo & 10. Felipe \\
\hline
\end{tabular}

\subsubsection{Nombres Femeninos}

\begin{tabular}{|c|c|c|}
\hline Estigmatizados & Prestigiosos & "Neutros" \\
\hline 1. Joscelyn (Yocelin) & 1. Martina & 1. Carla \\
\hline 2. Melany & 2. Sofía & 2. Carolina \\
\hline 3. Maryori & 3. Isidora & 3. Andrea \\
\hline 4. Yaritza & 4. Antonia & 4. Claudia \\
\hline 5. Sharon & 5. Josefa & 5. Alejandra \\
\hline 6. Shirley & 6. Ignacia & 6. Daniela \\
\hline 7. Nayareth & 7. María Paz & 7. Cecilia \\
\hline 8. Yamileth & 8. Agustina & 8. Paulina \\
\hline 9. Melody & 9. Julieta & 9. Fabiola \\
\hline 10. Britney & 10. Matilde & 10. Natalia \\
\hline
\end{tabular}




\subsection{Orden de los nombres dentro de la encuesta}

\subsubsection{Nombres masculinos}

\begin{tabular}{|c|c|c|c|c|c|}
\hline Cristóbal & Máximo & Jason & Héctor & Tomás & Jordan \\
\hline Alonso & Jeremy & Andrés & Rodrigo & Kevin & Joaquín \\
\hline Brian & Alejandro & Michael & Justin & Agustín & Ignacio \\
\hline Pablo & León & Claudio & Mauricio & Malcom & Sebastián \\
\hline Daniel & Byron & Harry & Felipe & Marcelo & Martín \\
\hline
\end{tabular}

\subsubsection{Nombres femeninos}

\begin{tabular}{|l|l|l|l|l|l|}
\hline Alejandra & Sharon & Fabiola & Ignacia & Martina & Claudia \\
\hline Isidora & Joselyn & Ma Paz & Carolina & Andrea & Melany \\
\hline Shirley & Sofía & Nayareth & Maryori & Cecilia & Carla \\
\hline Antonia & Agustina & Yamileth & Daniela & Britney & Julieta \\
\hline Josefa & Paulina & Matilde & Yaritza & Natalia & Melody \\
\hline
\end{tabular}

\section{ANÁLISIS Y RESULTADOS}

\subsection{Consideraciones previas}

La simbología empleada para señalar las expectativas de prestigio, estigmatización y neutralidad fue la siguiente:

- +: expectativa de valoración positiva del nombre (nombre prestigioso).

- +- : expectativa de valoración neutra del nombre (nombre no marcado).

- - : expectativa de valoración negativa del nombre (nombre estigmatizado).

En las páginas que siguen, a través de tablas, se presentan solamente los nombres que obtuvieron alguna votación. Después de cada tabla, se contienen algunos comentarios sobre aspectos prominentes que se desprenden de la información contenida en ellas. 


\subsection{Resultados versión sexo femenino}

\subsubsection{En relación con la pregunta:}

“Con cuáles de los siguientes nombres crees que tus amigos se reirían de ti o te molestarían?"

Tabla 1: Nombres con los cuales sujetos femeninos estiman que serían objeto de burla.

\begin{tabular}{|c|c|c|c|c|}
\hline $\mathrm{N}^{\circ}$ & Nombre & Cantidad & Porcentaje & Expectativa \\
\hline 1 & Shirley & 20 & $95,23 \%$ & - \\
\hline 2 & Yamileth & 20 & $95,23 \%$ & - \\
\hline 3 & Sharon & 19 & $90,47 \%$ & - \\
\hline 4 & Britney & 17 & $80,95 \%$ & - \\
\hline 5 & Maryori & 13 & $61,90 \%$ & - \\
\hline 6 & Melody & 12 & $57,14 \%$ & - \\
\hline 7 & Nayareth & 12 & $57,14 \%$ & - \\
\hline 8 & Yaritza & 10 & $47,61 \%$ & - \\
\hline 9 & Joselyn & 2 & $9,52 \%$ & - \\
\hline
\end{tabular}

\section{Observaciones}

Es interesante observar que los nombres más votados (Shirley y Yamileth), obtienen un altísimo 95,23\%, lo cual indica que estos nombres no fueron mencionados sólo por un sujeto femenino encuestado. Es interesante observar, también, que 9 de los 10 nombres con expectativa de valoración negativa recibieron votación. El único nombre con expectativa de valoración negativa que no recibió votación fue Melany, lo que se puede explicar por la alta concentración de votos en los otros nombres.

\subsubsection{En relación con la pregunta:}

“¿Con cuáles de los siguientes nombres piensas que tus amigos te considerarían más o serías más popular?” 
Tabla 2: Nombres con los cuales sujetos femeninos estiman que serían más populares.

\begin{tabular}{|c|c|c|c|c|}
\hline $\mathrm{N}^{\circ}$ & Nombre & Cantidad & Porcentaje & Expectativa \\
\hline 1 & Agustina & 14 & $66,66 \%$ & + \\
\hline 2 & Martina & 14 & $66,66 \%$ & + \\
\hline 3 & Josefa & 12 & $57,14 \%$ & + \\
\hline 4 & Antonia & 11 & $52,30 \%$ & + \\
\hline 5 & Sofía & 11 & $52,30 \%$ & + \\
\hline 6 & Isidora & 10 & $47,61 \%$ & + \\
\hline 7 & Matilde & 10 & $47,61 \%$ & + \\
\hline 8 & M Paz & 9 & $42,85 \%$ & + \\
\hline 9 & Ignacia & 7 & $33,33 \%$ & + \\
\hline 10 & Natalia & 6 & $28,57 \%$ & +- \\
\hline 11 & Julieta & 5 & $23,80 \%$ & + \\
\hline 12 & Alejandra & 5 & $23,80 \%$ & +- \\
\hline 13 & Paulina & 4 & $19,04 \%$ & +- \\
\hline 14 & Claudia & 3 & $14,28 \%$ & +- \\
\hline 15 & Andrea & 2 & $9,52 \%$ & +- \\
\hline 16 & Carolina & 2 & $9,52 \%$ & +- \\
\hline 17 & Carla & 1 & $4,76 \%$ & +- \\
\hline 18 & Daniela & 1 & $4,76 \%$ & +- \\
\hline 19 & Fabiola & 1 & $4,76 \%$ & + \\
\hline 20 & Melany & 1 & $4,76 \%$ & + \\
\hline
\end{tabular}

\section{Observaciones}

Al comparar esta tabla con la tabla 1, se puede observar que aquella que contiene los nombres con los cuales serían objeto de burla está mucho más cohesionada, pues contiene sólo 9 nombres, versus los 20 que contiene la tabla con los nombres prestigiosos. También es destacable que los nombres más votados en la tabla 2 (Agustina y Martina) corresponden a la preferencia de un $66.6 \%$ de los sujetos, muy distinto del $95.23 \%$ de los 2 nombres más votados de la tabla 1 . Por último, a diferencia de la tabla 1, en la tabla 2 hay un nombre con expectativa de valoración contraria (aunque sólo recibe un voto). 


\subsubsection{En relación con la pregunta:}

“¿Cuáles de estos nombres relacionas con un nivel social bajo?"

Tabla 3: Nombres que sujetos femeninos relacionan con un estrato social bajo.

\begin{tabular}{|c|c|c|c|c|}
\hline $\mathrm{N}^{\circ}$ & Nombre & Cantidad & Porcentaje & Expectativa \\
\hline 1 & Shirley & 19 & $90,47 \%$ & - \\
\hline 2 & Yamileth & 18 & $85,71 \%$ & - \\
\hline 3 & Britney & 15 & $71,42 \%$ & - \\
\hline 4 & Nayareth & 15 & $71,42 \%$ & - \\
\hline 5 & Maryori & 14 & $66,66 \%$ & - \\
\hline 6 & Sharon & 14 & $66,66 \%$ & - \\
\hline 7 & Yaritza & 14 & $66,66 \%$ & - \\
\hline 8 & Melody & 8 & $38,09 \%$ & - \\
\hline 9 & Joselyn & 4 & $19,04 \%$ & - \\
\hline 10 & Melany & 3 & $14,28 \%$ & + \\
\hline 11 & Matilde & 1 & $4,76 \%$ & - \\
\hline
\end{tabular}

\section{Observaciones}

Destaca aquí que el nombre más votado (Shirley), recibe un elevado $90,47 \%$ de las preferencias. Se puede apreciar, también, que todos los nombres con expectativa de valoración negativa fueron seleccionados por las encuestadas, y en las 10 primeras posiciones. Destaca, por último, la presencia de un nombre con expectativa de valoración contraria (Matilde), aunque sólo recibe un voto.

\subsubsection{En relación con la pregunta:}

“¿Cuáles de estos nombres relacionas con un nivel social alto?” 
Tabla 4: Nombres que sujetos femeninos relacionan con un estrato social alto.

\begin{tabular}{|c|c|c|c|c|}
\hline $\mathrm{N}^{\circ}$ & Nombre & Cantidad & Porcentaje & Expectativa \\
\hline 1 & Agustina & 19 & $90,47 \%$ & + \\
\hline 2 & Martina & 18 & $85,71 \%$ & + \\
\hline 3 & Isidora & 14 & $66,66 \%$ & + \\
\hline 4 & Antonia & 13 & $61,90 \%$ & + \\
\hline 5 & Sofía & 11 & $52,38 \%$ & + \\
\hline 6 & Ignacia & 10 & $47,61 \%$ & + \\
\hline 7 & Josefa & 10 & $47,61 \%$ & + \\
\hline 8 & Matilde & 10 & $47,61 \%$ & + \\
\hline 9 & M Paz & 7 & $33,33 \%$ & + \\
\hline 10 & Julieta & 6 & $28,57 \%$ & + \\
\hline 11 & Alejandra & 4 & $19,04 \%$ & +- \\
\hline 12 & Natalia & 2 & $9,52 \%$ & +- \\
\hline 13 & Carolina & 1 & $4,76 \%$ & + \\
\hline 14 & Paulina & 1 & $4,76 \%$ & + \\
\hline 15 & Melany & 1 & $4,76 \%$ & - \\
\hline
\end{tabular}

\section{Observaciones}

Al comparar esta tabla con la tabla 3, se puede observar que aquella que contiene los nombres que los sujetos relacionan con un estrato social bajo está más cohesionada, pues contiene sólo 11 nombres, versus los 15 que contiene la tabla con nombres que los sujetos asocian con un estrato social alto. Por otro lado, al igual que en la tabla 3, en la tabla 4 se observa que todos los nombres con la expectativa de valoración prevista (positiva, en este caso) fueron mencionados, y en las diez primeras posiciones. Destaca también la presencia de un nombre con expectativa de valoración contraria (Melany), aunque recibe sólo un voto.

\subsection{Resultados versión sexo masculino}

\subsubsection{En relación con la pregunta:}

“¿Con cuáles de los siguientes nombres crees que tus amigos se reirían de ti o te molestarían? 
Tabla 5: Nombres con los cuales sujetos masculinos estiman que serían objeto de burla.

\begin{tabular}{|c|c|c|c|c|}
\hline No & Nombre & Cantidad & Porcentaje & Expectativa \\
\hline 1 & Byron & 18 & $94,70 \%$ & - \\
\hline 2 & Brian & 16 & $84,21 \%$ & - \\
\hline 3 & Jason & 14 & $73,68 \%$ & - \\
\hline 4 & Malcon & 12 & $63,15 \%$ & - \\
\hline 5 & Justin & 11 & $57,89 \%$ & - \\
\hline 6 & Jeremy & 10 & $52,63 \%$ & - \\
\hline 7 & Jordan & 10 & $52,63 \%$ & - \\
\hline 8 & Michael & 8 & $42,10 \%$ & - \\
\hline 9 & Harry & 7 & $36,84 \%$ & + \\
\hline 10 & Kevin & 3 & $15,78 \%$ & +- \\
\hline 11 & León & 3 & $15,78 \%$ & +- \\
\hline 12 & Felipe & 1 & $5,26 \%$ & +- \\
\hline 13 & Marcelo & 1 & $5,26 \%$ & + \\
\hline 14 & Mauricio & 1 & $5,26 \%$ & + \\
\hline 15 & Cristóbal & 1 & $5,26 \%$ & $5,26 \%$ \\
\hline 16 & Martín & 1 & & - \\
\hline
\end{tabular}

\section{Observaciones}

Destaca aquí que el nombre más votado (Byron) recibe un altísimo 94.7\%, lo cual indica que sólo 1 sujeto no votó por este nombre. Se observa, también, que todos los nombres con expectativas de valoración negativa fueron seleccionados, y en los diez primeros lugares. Destaca, por último, la presencia de 3 nombres con expectativa de valoración contraria (León, Martín y Cristóbal). El más llamativo es León, pues, a diferencia de los otros, recibe tres votos.

\subsubsection{En relación con la pregunta:}

“¿Con cuáles nombres piensas que te considerarían más o serías más popular?” 
Tabla 6: Nombres con los cuales sujetos masculinos estiman que serían más populares.

\begin{tabular}{|c|c|c|c|c|}
\hline $\mathrm{N}^{\circ}$ & Nombre & Cantidad & Porcentaje & Expectativa \\
\hline 1 & Cristóbal & 11 & $57,89 \%$ & + \\
\hline 2 & Sebastián & 10 & $52,63 \%$ & + \\
\hline 3 & Alonso & 9 & $47,36 \%$ & + \\
\hline 4 & Rodrigo & 9 & $47,36 \%$ & +- \\
\hline 5 & Ignacio & 7 & $36,84 \%$ & + \\
\hline 6 & Joaquín & 7 & $36,84 \%$ & + \\
\hline 7 & Tomás & 7 & $36,84 \%$ & + \\
\hline 8 & Pablo & 7 & $36,84 \%$ & +- \\
\hline 9 & Martín & 6 & $31,57 \%$ & + \\
\hline 10 & Daniel & 6 & $31,57 \%$ & +- \\
\hline 11 & Felipe & 5 & $26,31 \%$ & +- \\
\hline 12 & Marcelo & 5 & $26,31 \%$ & + \\
\hline 13 & Máximo & 4 & $21,05 \%$ & + \\
\hline 14 & Agustín & 3 & $15,78 \%$ & +- \\
\hline 15 & Alejandro & 3 & $15,78 \%$ & +- \\
\hline 16 & Andrés & 3 & $15,78 \%$ & +- \\
\hline 17 & Claudio & 3 & $15,78 \%$ & + \\
\hline 18 & Héctor & 3 & $15,78 \%$ & + \\
\hline 19 & Justin & 2 & $10,52 \%$ & $5,26 \%$ \\
\hline 20 & León & 1 & $5,26 \%$ & +- \\
\hline 21 & Mauricio & 1 & & + \\
\hline
\end{tabular}

\section{Observaciones}

Al comparar esta tabla con la tabla 5, se puede observar que aquella que contiene los nombres con los que serían objeto de burla es más compacta, pues contiene 16 nombres, versus los 21 que contiene la tabla con los nombres que los harían más populares. No sorprende, entonces, que, al igual que la tabla 5, en la tabla 6 los 10 nombres con la expectativa de valoración prevista (positiva, en este caso) fueran mencionados.

Nótese, además, que el nombre más votado (Cristóbal) corresponde a la preferencia de 11 sujetos (57.89\%), versus los 18 votos (94.7\%) del nombre más votado de la tabla 5 . Valga señalar que, en alguna medida, sorprende la alta votación 
del nombre Rodrigo (47.36\%), pues respecto de él se tenía una expectativa de valoración neutra. Destaca, por último, la presencia de un nombre con expectativa de valoración contraria (Justin), el cual recibió dos votos.

\subsubsection{En relación con la pregunta:}

“Cuáles de estos nombres relacionas con un nivel social bajo?”

Tabla 7: Nombres que sujetos masculinos relacionan con un estrato social bajo.

\begin{tabular}{|c|c|c|c|c|}
\hline $\mathrm{N}^{\circ}$ & Nombre & Cantidad & Porcentaje & Expectativa \\
\hline 1 & Byron & 19 & $100 \%$ & - \\
\hline 2 & Jason & 16 & $84,2 \%$ & - \\
\hline 3 & Brian & 12 & $63,1 \%$ & - \\
\hline 4 & Jeremy & 12 & $63,1 \%$ & - \\
\hline 5 & Jordan & 11 & $57,8 \%$ & - \\
\hline 6 & Justin & 11 & $57,8 \%$ & - \\
\hline 7 & Malcom & 10 & $52,6 \%$ & - \\
\hline 8 & Kevin & 6 & $31,7 \%$ & - \\
\hline 9 & Michael & 6 & $31,7 \%$ & + \\
\hline 10 & Harry & 5 & $26,3 \%$ & + \\
\hline 11 & León & 2 & $10,5 \%$ & +- \\
\hline 12 & Héctor & 1 & $5,2 \%$ & + \\
\hline 13 & Mauricio & 1 & $5,2 \%$ & $+2 \%$ \\
\hline 14 & Máximo & 1 & $5,2 \%$ & + \\
\hline 15 & Cristóbal & & & + \\
\hline
\end{tabular}

\section{Observaciones}

Destaca de manera prominente el hecho de que el nombre Byron reciba el voto de todos y cada uno de los sujetos masculinos encuestados. Además, se observa que las expectativas de valoración se cumplen, pues los diez primeros nombres son aquellos que tenían una expectativa de valoración negativa. Se observa, por último, que ocurren tres nombres con expectativas de valoración contraria (León, Máximo y Cristóbal), aunque este hecho no debe sobredimensionarse, pues estos nombres no reciben más de dos votos. 


\subsubsection{En relación con la pregunta:}

“¿Cuáles de estos nombres relacionas con un nivel social alto?”

Tabla 8: Nombres que sujetos masculinos relacionan con un estrato social alto.

\begin{tabular}{|c|c|c|c|c|}
\hline $\mathrm{N}^{\circ}$ & Nombre & Cantidad & Porcentaje & Expectativas \\
\hline 1 & Agustín & 10 & $52,6 \%$ & + \\
\hline 2 & Alonso & 10 & $52,6 \%$ & + \\
\hline 3 & Joaquín & 10 & $52,6 \%$ & + \\
\hline 4 & León & 10 & $52,6 \%$ & + \\
\hline 5 & Tomás & 9 & $47,3 \%$ & + \\
\hline 6 & Alejandro & 9 & $47,3 \%$ & +- \\
\hline 7 & Cristóbal & 8 & $42,1 \%$ & + \\
\hline 8 & Martín & 8 & $42,1 \%$ & + \\
\hline 9 & Sebastián & 8 & $42,1 \%$ & + \\
\hline 10 & Ignacio & 7 & $36,8 \%$ & + \\
\hline 11 & Máximo & 5 & $26,3 \%$ & + \\
\hline 12 & Daniel & 4 & $21 \%$ & +- \\
\hline 13 & Andrés & 3 & $15,7 \%$ & + \\
\hline 14 & Héctor & 2 & $10,5 \%$ & + \\
\hline 15 & Mauricio & 2 & $10,5 \%$ & + \\
\hline 16 & Pablo & 2 & $10,5 \%$ & + \\
\hline 17 & Rodrigo & 2 & $10,5 \%$ & +- \\
\hline 18 & Claudio & 1 & $5,2 \%$ & +- \\
\hline 19 & Felipe & 1 & $5,2 \%$ & + \\
\hline 20 & Harry & 1 & $5,2 \%$ & + \\
\hline 21 & Justin & 1 & $5,2 \%$ & + \\
\hline 22 & Kevin & 1 & & + \\
\hline
\end{tabular}

\section{Observaciones}

Al comparar esta tabla con la tabla 7 , se puede observar que aquella que contiene los nombres que los sujetos asocian con un estrato social bajo está más cohesionada, pues contiene 15 nombres, versus los 22 que contiene la tabla con nombres que se asocian con un estrato social alto. 
Es interesante destacar, también, que el porcentaje de los nombres más votados de la tabla 8 (Agustín y Alonso), corresponde a la votación de 10 sujetos (52.6\%), versus los 19 (100\%) que votaron por el nombre con expectativa de valoración negativa Byron.

Se observa, por último, que la expectativa de valoración se cumple cabalmente en los cinco primeros nombres. Luego, sorprende la aparición del nombre Alejandro (con un 47,3\%, equivalente a 9 votos), pues respecto de él se tenía una expectativa de valoración neutra y, sin embargo, ocupa una posición prominente. En las cinco posiciones siguientes aparecen Cristóbal, Martín, Sebastián, Ignacio y Máximo, con lo que se retoman las expectativas de valoración positiva.

\section{CONCLUSIONES}

Podemos afirmar, en primer lugar, que los objetivos que nos propusimos se cumplieron, pues se determinó el grado de prestigio y estigmatización que 40 sujetos, con un nivel educacional superior (universitario), otorgan a los nombres propios contenidos en el instrumento. Así, se logró establecer el grado de popularidad o desprestigio que los individuos de la muestra atribuyen a los nombres seleccionados; a la vez que se estableció la asociación de estos con un estrato sociocultural alto o bajo.

Visto todo lo anterior, se puede concluir, en primer lugar, que existe una alta conciencia de norma por parte de los encuestados y encuestadas. En efecto, la ocurrencia de una alta concentración de las preferencias y la alta cantidad de votos recibida por algunos nombres, especialmente en las preguntas asociadas con estigmatización, nos informan que los sujetos poseen una alta apropiación de los códigos sociales asociados con prestigio y discriminación.

También se puede constatar que las votaciones de las mujeres son las que concuerdan en mayor medida con las expectativas otorgadas en el diseño del instrumento. Esto corrobora lo propuesto por investigadores como Tassara (1992), cuando afirma que los jóvenes y las mujeres son los grupos que de manera más drástica emiten juicios sobre el uso de la lengua. 
Además, es destacablequelacantidad denombresvotados porlosencuestados y encuestadas es siempre inferior en las preguntas relativas a estigmatización (lo que se asocia con la alta concentración de votos en determinados nombres). Esto revela que la elección de un nombre hace emerger en estos sujetos las distintas asunciones culturales que han internalizado en su comunidad de habla.

Una reflexión final cabe aquí. Así como el estudio de Figueroa (2011) muestra cómo los sujetos establecieron una asociación directa entre determinados sonidos y el estrato social de quienes los emiten, nuestra investigación muestra cómo en el ámbito de los nombres propios sucede algo similar, pues los sujetos asocian claramente algunos de ellos con popularidad/estrato alto y desprestigio/ estrato bajo. Así las cosas, nuestros datos contribuyen con más evidencias que respaldan la hipótesis de que ciertos sectores de nuestra población segregan a otros sectores de la población, entre otros factores, por aspectos vinculados con el lenguaje.

Como posibles proyecciones, se plantea la posibilidad de utilizar otros nombres propios, con el fin de confirmar, matizar o refutar las tendencias aquí presentadas. Además, se podría comparar la conciencia de norma (socio) lingüística en diversos grupos etarios. Una posibilidad consistiría en encuestar a grupos de adolescentes y adultos mayores; otra posibilidad consistiría en realizar un estudio donde se comparen las respuestas de niños de cuarto año básico, con las de niños de octavo año básico y adolescentes que estén cursando cuarto año de enseñanza media, para rastrear a qué edad comienza a aparecer el concepto de prestigio/ estigmatización.

Otra proyección posible consiste en incluir la variable diatópica, aplicando este instrumento, por ejemplo, en un contexto urbano-rural. Así, se podría aplicar la encuesta a jóvenes de un liceo de Concepción (urbano) y a jóvenes de un liceo de Mulchén (rural).

Un imperativo pendiente consiste en analizar las respuestas correspondientes a las otras preguntas contenidas en el instrumento ("imagina personas que lleven estos nombres y describe: a) su probable apariencia física, b) sus eventuales gustos, c) dónde vivirían y d) dónde estudiarían”, y “¿cuáles crees tú que son los factores que influyen para que un nombre sea considerado 'bonito' o 'feo'?"). 
Por último, sería interesante realizar un estudio a nivel hispanoamericano, en relación con prestigio y estigmatización de nombres propios en otros países de habla castellana.

\section{REFERENCIAS}

Fasold, Ralph. La Sociolingüistica de la sociedad. Introducción a la sociolingüistica. Madrid: Visor Libros, 1996.

Figueroa, Mauricio. "El eje oclusión/fricción en el sistema sociofónico del castellano de Chile”. Tesis para optar al grado de Magíster en Lingüística. Chile: Universidad de Concepción, 2011.

Millán, Macarena y Rodríguez, Claudia. "Prestigio y estigmatización de 30 nombres propios en 44 sujetos de estrato sociocultural bajo”. Tesis para optar al grado de Licenciado en Educación con Mención en Español. Chile: Universidad de Concepción, 2011.

Sáez, Leopoldo. El español de Chile en las postrimerías del siglo XX. Santiago de Chile: Universidad de Santiago, 1999.

Salamanca, Gastón. "Apuntes sociolingüísticos sobre la presencia de argentinismos en el léxico del español de Chile”, Atenea 502 (2010): 125-149.

Salamanca, Gastón y Ramírez, Ariella. "Argentinismos en el léxico del español de Chile: nuevas evidencias", Atenea, por aparecer.

Salamanca, Gastón y Valverde, Anita. "Prestigio y estigmatización en variantes anteriorizadas y posteriorizadas de las vocales del español de Chile”, Literatura y Lingüistica 20 (2009): 125-140.

Salas, Adalberto. "Aymara mapuche ¿lenguas o dialectos? En torno a la valoración social de los vernáculos chilenos”, Atenea 473 (1996): 25-37.

Silva-Corvalán, Carmen. Sociolingüistica y Pragmática del español. Washington D.C.: Georgetown University Press, 2001.

Tassara, Gilda. "Actitudes lingüísticas ante la variación de /ĉl”, Revista de Lingüística Teórica y aplicada 30 (1992): 263-271. 
Valdivieso, Humberto. "Prestigio y estigmatización: factor determinante en la enseñanza institucionalizada de la lengua materna", RLA. Revista de Lingüistica Teórica y Aplicada 21 (1983): 137-142.

\section{ANEXOS}

Preguntas efectivamente consideradas para el análisis

a) Versión para sexo femenino

1 a) ¿Con cuáles de los siguientes nombres crees que tus amigos se reirían de ti o te molestarían?, ¿y con cuáles piensas que te considerarían más o serías más popular? Selecciona 6 para cada categoría. Marca aquéllos con los cuales crees que serías bien vista con un $\checkmark$ y aquéllos con los cuales crees que se reirían de ti, con una $\mathrm{X}$.

\begin{tabular}{|l|l|l|l|l|l|l|l|l|l|}
\hline Alejandra & Sharon & & Fabiola & & Ignacia & & Martina & Claudia & \\
\hline Isidora & Joselyn & & Ma Paz & Carolina & & Andrea & Melany & \\
\hline Shirley & Sofía & Nayareth & Maryori & Cecilia & Carla & \\
\hline Antonia & Agustina & Yamileth & Daniela & Britney & Julieta & \\
\hline Josefa & Paulina & Matilde & Yaritza & Natalia & Melody & \\
\hline
\end{tabular}

2 a) ¿Cuáles de estos nombres relacionas con un nivel social alto y cuáles con un nivel social bajo? Selecciona 6 y ubícalos en cada columna según corresponda.

Nombres de mujeres:

\begin{tabular}{|l|l|l|l|l|l|}
\hline Alejandra & Sharon & Fabiola & Ignacia & Martina & Claudia \\
\hline Isidora & Joselyn & $\mathrm{M}^{\mathrm{a}}$ Paz & Carolina & Andrea & Melany \\
\hline Shirley & Sofía & Nayareth & Maryori & Cecilia & Carla \\
\hline Antonia & Agustina & Yamileth & Daniela & Britney & Julieta \\
\hline Josefa & Paulina & Matilde & Yaritza & Natalia & Melody \\
\hline
\end{tabular}




\begin{tabular}{|l|l|}
\hline \multicolumn{1}{|c|}{ NIVEL ALTO } & \multicolumn{1}{c|}{ NIVEL BAJO } \\
\hline 1. & 1. \\
\hline 2. & 2. \\
\hline 3. & 3. \\
\hline 4. & 4. \\
\hline 5. & 5. \\
\hline 6. & 6. \\
\hline
\end{tabular}

3. a) A partir de la lista de nombres que a continuación aparecen, selecciona 6 y ordena según tu preferencia para colocarles a tus hijos, 3 para hombre y 3 para mujer. Además elige 6 nombres que NO les pondrías a tus hijos, 3 para hombre y 3 para mujer.

NOMBRES PARA HOMBRE

\begin{tabular}{|l|l|l|l|l|l|}
\hline Cristóbal & Máximo & Jason & Héctor & Tomás & Jordan \\
\hline Alonso & Jeremy & Andrés & Rodrigo & Kevin & Joaquín \\
\hline Malcom & Alejandro & Michael & Justin & Agustín & Ignacio \\
\hline Pablo & León & Claudio & Mauricio & Brian & Sebastián \\
\hline Daniel & Byron & Harry & Felipe & Marcelo & Martín \\
\hline
\end{tabular}

NOMBRES PARA MUJER

\begin{tabular}{|l|l|l|l|l|l|}
\hline Alejandra & Sharon & Fabiola & Ignacia & Martina & Claudia \\
\hline Isidora & Joselyn & Ma Paz & Carolina & Andrea & Melany \\
\hline Shirley & Sofía & Nayareth & Maryori & Cecilia & Carla \\
\hline Antonia & Agustina & Yamileth & Daniela & Britney & Julieta \\
\hline Josefa & Paulina & Matilde & Yaritza & Natalia & Melody \\
\hline
\end{tabular}

\section{IMPORTANTE:}

Debes poner el nombre que más te gusta en el No 1 de la primera tabla, y el que menos te gusta en el No 1 de la segunda tabla, y continuar de forma decreciente en las casillas que siguen hasta completar los 12 nombres. 


\begin{tabular}{|l|l|}
\hline \multicolumn{2}{|c|}{ NOMBRES QUE PREFERIRÍA } \\
\hline \multicolumn{1}{|c|}{ Si fuese hombre } & \multicolumn{1}{c|}{ Si fuese mujer } \\
\hline 1. & 1. \\
\hline 2. & 2. \\
\hline 3. & 3. \\
\hline
\end{tabular}

\begin{tabular}{|l|l|}
\hline \multicolumn{2}{|c|}{ NOMBRES QUE NUNCA ELIGIRÍA } \\
\hline \multicolumn{1}{|c|}{ Si fuese hombre } & \multicolumn{1}{c|}{ Si fuese mujer } \\
\hline 1. & 1. \\
\hline 2. & 2. \\
\hline 3. & 3. \\
\hline
\end{tabular}

b) Versión para sexo masculino

1 a) ¿Con cuáles de los siguientes nombres crees que tus amigos se reirían de ti o te molestarían?, ¿y con cuáles piensas que te considerarían más o serías más popular? Selecciona 6 para cada categoría. Marca aquéllos con los cuales crees que serías bien visto con un $\checkmark$ y aquéllos con los cuales crees que se reirían de ti, con una $\mathrm{X}$.

\begin{tabular}{|c|c|c|c|c|c|c||}
\hline Cristóbal & Máximo & Jason & Héctor & Tomás & Jordan \\
\hline Alonso & Jeremy & Andrés & Rodrigo & Kevin & Joaquín \\
\hline Malcom & Alejandro & Michael & Justin & Agustín & Ignacio \\
\hline Pablo & León & Claudio & Mauricio & Brian & Sebastián \\
\hline Daniel & Byron & Harry & Felipe & Marcelo & Martín \\
\hline
\end{tabular}

2 a) ¿Cuáles de estos nombres relacionas con un nivel social alto y cuáles con un nivel social bajo? Selecciona 6 y ubícalos en cada columna según corresponda.

Nombres de hombres:

\begin{tabular}{|c|c|c|c|c|c|c|c|}
\hline Cristóbal & Daniel & León & Michel & Rodrigo & Tomás & Marcelo & Sebastián \\
\hline Alonso & Máximo & Byron & Claudio & Justin & Kevin & Jordan & Martín \\
\hline Malcom & Jeremy & Jason & Harry & Mauricio & Agustín & Joaquín & \\
\hline Pablo & Alejandro & Andrés & Héctor & Felipe & Brian & Ignacio & \\
\hline
\end{tabular}




\begin{tabular}{|l|l|}
\hline \multicolumn{1}{|c|}{ NIVEL ALTO } & \multicolumn{1}{c|}{ NIVEL BAJO } \\
\hline 1. & 1. \\
\hline 2. & 2. \\
\hline 3. & 3. \\
\hline 4. & 4. \\
\hline 5. & 5. \\
\hline 6. & 6. \\
\hline
\end{tabular}

3 a) A partir de la lista de nombres que a continuación aparecen, selecciona 6 y ordena según tu preferencia para colocarles a tus hijos, 3 para hombre y 3 para mujer. Además elige 6 nombres que NO les pondrías a tus hijos, 3 para hombres y 3 para mujer.

NOMBRES PARA HOMBRE

\begin{tabular}{|l|l|l|l|l|l|}
\hline Cristóbal & Máximo & Jason & Héctor & Tomás & Jordán \\
\hline Alonso & Jeremy & Andrés & Rodrigo & Kevin & Joaquín \\
\hline Malcom & Alejandro & Michael & Justin & Agustín & Ignacio \\
\hline Pablo & León & Claudio & Mauricio & Brian & Sebastián \\
\hline Daniel & Byron & Harry & Felipe & Marcelo & Martín \\
\hline
\end{tabular}

NOMBRES PARA MUJER

\begin{tabular}{|l|l|l|l|l|l|}
\hline Alejandra & Sharon & Fabiola & Ignacia & Martina & Claudia \\
\hline Isidora & Joselyn & $\mathrm{M}^{\mathrm{a}}$ Paz & Carolina & Andrea & Melany \\
\hline Shirley & Sofia & Nayareth & Maryori & Cecilia & Carla \\
\hline Antonia & Agustina & Yamileth & Daniela & Britney & Julieta \\
\hline Josefa & Paulina & Matilde & Yaritza & Natalia & Melody \\
\hline
\end{tabular}




\section{IMPORTANTE:}

Debes poner el nombre que más te gusta en el No 1 de la primera tabla, y el que menos te gusta en el No 1 de la segunda tabla, y continuar de forma decreciente en las casillas que siguen hasta completar los 12 nombre

\begin{tabular}{|l|l|}
\hline \multicolumn{2}{|c|}{ NOMBRES QUE PREFERIRÍA } \\
\hline \multicolumn{1}{|c|}{ Si fuese hombre } & \multicolumn{1}{c|}{ Si fuese mujer } \\
\hline 1. & 1. \\
\hline 2. & 2. \\
\hline 3. & 3. \\
\hline
\end{tabular}

\begin{tabular}{|l|l|}
\hline \multicolumn{2}{|c|}{ NOMBRES QUE NUNCA ELEGIRÍA } \\
\hline \multicolumn{1}{|c|}{ Si fuese hombre } & \multicolumn{1}{c|}{ Si fuese mujer } \\
\hline 1. & 1. \\
\hline 2. & 2. \\
\hline 3. & 3. \\
\hline
\end{tabular}

